\title{
Advances in Assessing Preoperative Liver Function with Gd-EOB-DTPA Dynamic Contrast Enhanced MRI
}

\author{
Juan Li1, Bing Wan², Sibin Liü ${ }^{2 *}$ \\ ${ }^{1}$ Department of Medicine, Yangtze University, Jingzhou, China \\ ${ }^{2}$ Radiology Department, Jingzhou Central Hospital, Jingzhou, China \\ Email: quanquanlii@163.com, *liusibin9159@qq.com
}

How to cite this paper: Li, J., Wan, B. and Liu, S.B. (2019) Advances in Assessing Preoperative Liver Function with Gd-EOB-DTPA Dynamic Contrast Enhanced MRI. Yangtze Medicine, 3, 32-42.

https://doi.org/10.4236/ym.2019.31004

Received: December 26, 2018

Accepted: March 19, 2019

Published: March 22, 2019

Copyright $\odot 2019$ by author(s) and Scientific Research Publishing Inc. This work is licensed under the Creative Commons Attribution International License (CC BY 4.0).

http://creativecommons.org/licenses/by/4.0/

\begin{abstract}
Liver cancer is the common malignant tumor in China and current treatment is based on surgery. However, liver function of many liver cancer patients is impaired before surgery, so there's a high possibility of occurrence of liver failure after the tumor resection. Therefore, it's necessary to accurately evaluate liver function before surgery. Currently, clinical methods are mostly limited to assess the function of overall liver. But the application of hepatocyte-specific contrast agent-gadolinium ethoxybenzyl diethylenetriamine pentaacetic acid (Gd-EOB-DTPA) makes it possible to assess the function of local liver segment accurately. This paper reviewed the progress of using Gd-EOB-DTPA dynamic contrast enhanced magnetic resonance imaging (MRI) to assess liver function preoperatively, such as parameters selection for liver function assessment, clinical factors affecting Gd-EOB-DTPA enhanced MRI and so on.
\end{abstract}

\section{Keywords}

Liver Function, Gd-EOB-DTPA, MRI, Dynamic Contrast Enhancement

\section{Introduction}

Liver cancer is the common malignant tumor in China, and ranks third in all the tumor incidence [1] [2]. Actually, some patients with liver cancer are not suitable for liver transplantation, and for the limited number of transplant recipients, the current treatment is still mainly based on surgical resection in China. However, liver cancer patients are usually accompanied with chronic hepatitis, schistosomiasis liver disease, liver cirrhosis and other chronic liver diseases [3]. In 
addition to the tumor lesions, chronic liver diseases also cause varying degrees damage to the liver, resulting in insufficient liver reserve capacity. Unfortunately the latter is an important reason for the liver failure [4]. Therefore, accurately assessment of liver function preoperative is helpful to reduce the incidence of postoperative liver failure.

Liver has many functions such as metabolism, secretion, excretion, and detoxification; correspondingly there're many ways to assess liver function. At present, clinical commonly used methods are limited in assessing overall liver function, not accurately assessing local segment liver function [5] [6]. However, in conditions such as portal embolization or unilateral cholestasis, damages often occur in local segment. The assessment methods of overall liver function do not truly reflect the function at a specific site. Gadolinium ethoxybenzyl diethylenetriamine pentaacetic acid (Gd-EOB-DTPA, Prinovist, Schering, Berlin, Germany) has the double characteristics of extracellular contrast agent and intracellular contrast agent, and is able to evaluate liver function in a specific site of local liver segment, thus becoming a research hotspot in recent years.

Gd-EOB-DTPA is a new kind of multifunctional magnetic resonance contrast agent produced by Bayer Schering Pharma AG in 1992, and it has undergone I, II, and III clinical trials since then [7]. Lastly, it was officially listed in China in July 2011 [8]. Many studies have indicated that Gd-EOB-DTPA enhanced MRI has high accuracy in detection and characterization of focal liver lesions as yet. It has the ability to distinguish between benign and malignant liver diseases such as hepatic cell carcinoma (HCC), regenerative nodule ( $\mathrm{RN})$, dysplastic nodule (DN) and liver metastases [8] [9]. Not only that, Gd-EOB-DTPA enhanced MR cholangiography can be used for the diagnosis of diseases such as biliary obstruction and gallstones [10]. However this review will focus on the progress of Gd-EOB-DTPA used in liver function assessment.

\section{Common Methods of Liver Function Assessment}

\subsection{Blood Parameters}

Measuring blood parameters is the most basic and widely used method, and the commonly concerned parameters include albumin(Alb), bilirubin, alanine aminotransferase (ALT), aspartate aminotransferase (AST), alkaline phosphatase (ALP), glutamyl transpeptidase (GGT) and proenzyme time (PT). This method is easy to perform and can initially assess liver function, but these indicators only reflect the degree of liver damage, not directly assess liver function [11].

\subsection{Comprehensive Scoring Systems}

The most widely used methods in clinical are the Child-Turcotte-Pugh (CTP) classification and the model for end-stage liver disease (MELD) score. Their drawbacks are that they are not suitable for non-cirrhosis patients, and the liver function difference between patients of the same level can be very large [12]. That's to say, patients with the lowest level of liver damage may also experience 
serious complications such as liver failure after the hepatectomy.

\subsection{Quantitative Experiments of Liver Function}

The always used method is indocyanine green (ICG) test which is the most accurate technique for assessing liver function now [13]. But during the test, the effects of liver on drug intake and metabolism are considered, while the effects of hepatic blood flow, bile and liver material exchange on drugs are ignored. More importantly, the test is also an evaluation of overall liver function with limited reference value in clinical practice [14].

\subsection{Liver Volume Measurement}

It mainly includes methods of measuring the physical volume of liver by using imaging techniques such as computed tomography (CT) and MRI, and measuring functional volume of liver by using nuclear medicine technology which is represented by $99 \mathrm{mTc}$ Gamma Scintigraphy (99mTc-GSA) [15] [16]. Comparing these two methods, measuring functional volume is better because the liver's physical volume and actual function are always not in consistence. But, considering the complexity of operation and high price of radioactive elements, it's difficult to popularize in clinical practice [17] (Table 1).

Table 1. Comparison of common methods for liver function assessment. These five methods in are available for liver function assessment and each has advantages and disadvantages. However, the first three methods are only used for overall liver function assessment, and the latter two methods can be used for local liver function assessment. Comparing the latter two methods, Gd-EOB-DTPA enhanced MRI is more convenient to operate.

\begin{tabular}{|c|c|c|c|c|}
\hline Method & Advantage & Disadvantage & Clinical application & Example \\
\hline Blood parameters & $\begin{array}{l}\text { Basic assessment; } \\
\text { Easy to operate; } \\
\text { Cheap }\end{array}$ & $\begin{array}{l}\text { Overall liver assessment; } \\
\text { Individual difference; } \\
\text { Only reflect liver damage }\end{array}$ & $\begin{array}{l}\text { Most basic and } \\
\text { widely used }\end{array}$ & $\begin{array}{l}\text { Alb; bilirubin; } \\
\text { ALT; AST; PT }\end{array}$ \\
\hline Comprehensive scoring systems & $\begin{array}{l}\text { Basic assessment; } \\
\text { Non-individual difference; } \\
\text { Easy to operate; } \\
\text { Cheap }\end{array}$ & $\begin{array}{l}\text { Overall liver assessment; } \\
\text { Not suitable for } \\
\text { non-cirrhosis patients }\end{array}$ & $\begin{array}{l}\text { Most basic and } \\
\text { widely used }\end{array}$ & $\begin{array}{l}\text { CTP-classification; } \\
\text { MELD score }\end{array}$ \\
\hline Quantitative experiments & $\begin{array}{l}\text { Accurate assessment; } \\
\text { Relatively easy to operation; } \\
\text { Relatively cheap }\end{array}$ & $\begin{array}{l}\text { Overall liver assessment } \\
\text { only consider the effects of liver } \\
\text { on drug intake and metabolism }\end{array}$ & Frequently used & ICG test \\
\hline Liver volume measurement & $\begin{array}{l}\text { Accurate assessment; } \\
\text { Local segment assessment; } \\
\text { Provide anatomical } \\
\text { and functional information }\end{array}$ & $\begin{array}{l}\text { Complex to operate; } \\
\text { Radioactivity expensive }\end{array}$ & $\begin{array}{l}\text { Used only for } \\
\text { academic research }\end{array}$ & 99mTc-GSA \\
\hline$G d-E O B-D T P A$ enhanced $M R I$ & $\begin{array}{l}\text { Accurate assessment; } \\
\text { Local segment assessment }\end{array}$ & $\begin{array}{l}\text { Evaluation parameters and } \\
\text { standards have not yet reached } \\
\text { the unified standard; } \\
\text { Relatively expensive }\end{array}$ & $\begin{array}{l}\text { Used only for } \\
\text { academic research }\end{array}$ & $\begin{array}{l}\text { Gd-EOB-DTPA } \\
\text { enhanced MRI }\end{array}$ \\
\hline
\end{tabular}




\section{Overview and Advantages of Gd-EOB-DTPA}

\subsection{Pharmacokinetics of Gd-EOB-DTPA}

Gd-EOB-DTPA is a hepatocyte target contrast agent adding a unique lipophilic EOB group, which makes the hepatocyte uptake rate as high as $50 \%$, that can significantly increase the $\mathrm{T} 1$ relaxation rate and shorten the $\mathrm{T} 1$ relaxation time [9]. Most importantly, Gd-EOB-DTPA has both the characteristics of a non-specific extracellular contrast agent and the specific intracellular contrast agent [18]. Gd-EOB-DTPA enters hepatocytes through the organic anion-transporting polypeptide 1 (OATP1) transporter on the hepatocyte membrane, so in the severely damaged liver, Gd-EOB-DTPA cannot be concentrated in hepatocytes due to the lack of the OATP1 transporter. As a result, the relevant area is in low development or cannot be developed, thereby exhibiting a low signal and being distinguishable from a high signal in a normal area [19].

\subsection{Advantages of Gd-EOB-DTPA}

Comparing with other hepatobiliary-specific agents, for example gadopentetate dimeglumine (Gd-DTPA), the advantages of Gd-EOB-DTPA mainly in: 1) Gd-EOB-DTPA has dual channels of excretion that can be excreted by the biliary tract and kidneys at the same time, and the ratio of two ways is about $50 \%$ [20]. That is to say, when there is an excretion disorder in one of the two ways, it can be compensated by another way; 2) To achieve the same degree of development, Gd-EOB-DTPA requires only one quarter of the amount of Gd-DTPA [21]; 3) Compared with Gd-DTPA, Gd-EOB-DTP can reach developmental requirements $20 \mathrm{~min}$ after injection instead of $40 \mathrm{~min}$, thus significantly reducing inspection time; (4) Gd-EOB-DTPA is safer because it causes less adverse reactions [22] (Table 2).

\section{The Setting of Flip Angle and Scan Time during Examination}

In general, hepatocytes start to taking up the contrast agent after 1.5 min of contrast injection, and 20 min after Gd-EOB-DTPA enhancement is defined as the "standard" hepatobiliary phase (HBP). Recently, there're many arguments about the choice of scanning parameters, especially for flip angle (FA) and start scan time of HBP.

Table 2. Difference between Gd-EOB-DTPA and Gd-DTPA. Gd-EOB-DTPA has many advantages over other hepatobiliary-specific agents (such as Gd-DTPA), making it more suitable for clinical applications.

\begin{tabular}{ccc}
\hline Contrast agent & Gd-EOB-DTPA & Gd-DTPA \\
\hline Excretion & Liver and kidneys & Only kidneys \\
Dosage & $0.025 \mathrm{mmol} / \mathrm{kg}$ & $0.1 \mathrm{mmol} / \mathrm{kg}$ \\
Examination time & $20 \mathrm{~min}$ & $40 \mathrm{~min}$ \\
Adverse reactions & less & more \\
\hline
\end{tabular}


Zhi-Peng zhou et al. [23] compared 10-min HBP and 20-min HBP images of 103 patients after Gd-EOB-DTPA enhancement, founding that the 10 -min HBP point was a viable option for quantitatively assessing liver function while reducing examination time. Inhwan Jeon et al. [24] made imaging experiments using three different parameters: 10 min-FA30, 20 min-FA10, and $20 \mathrm{~min}-\mathrm{FA} 30$, and results showed that diagnostic performance of 10 min-FA30 was higher than that of 20 min-FA10 and 20 min-FA30, regardless of whether the liver function was in the compensated or decompensated period. These indicate that $10-\mathrm{min}$ after the contrast agent injection, increasing the FA can be used to perform HBP imaging without considering the liver function. However, Okada et al. [25] reported that by analying FA of 9 different values, the image quality obtained by FA 10 - 20 was the best, and when FA > 20, the image quality would be degraded. Similarly, Cui Enming et al. [26] believed that although increasing FA could significantly improve image contrast, but it would inevitably reduce the signal to noise ratio (SNR) of images, leading to missed diagnosis of small lesions.

\section{Parameters Selection for Liver Function Assessment}

Lan Lianjun et al. [27] pointed out that parameters of Gd-EOB-DTPA enhanced MRI for assessing liver function can be divided into four categories: 1) T1 relaxation; 2) $\mathrm{T} 2^{*}$ relaxation time reduction rate; 3) biliary enhancement; 4) Gd-EOB-DTPA MRI perfusion imaging. Among them, T1 relaxation is the most widely used parameter. The specific evaluation indicators include relative enhancement (RE), hepatocellular uptake index (HUI), liver uptake rate and intake fraction, T1 relaxation time and T1 map.RE is calculated according to the degree of liver parenchymal enhancement before and after Gd-EOB-DTPA enhanced MRI scan (SIpre, SIpost), and the specific formula is:

$$
\operatorname{RE}(\%)=[(\text { SIpost }- \text { SIpre }) / \text { SIpre }] \text {; }
$$

HUI is based on the measurement of liver and spleen signal intensity (L20, S20) and liver volume (VL), and then calculated according to the formula HUI = VL[(L20/S20) - 1]; Liver uptake rate and uptake fraction, T1 relaxation time and T1 map are mainly based on the T1 value of the liver before and after enhancement [27]. No matter evaluation parameters based on SI or T1 values, they are statistically well correlated with CTP classification, MELD score, ICG test and other evaluation methods, thus confirming the accuracy of these evaluation parameters [17] [28] [29].

Michael Haimerl et al. [30] performed Gd-EOB-DTPA enhanced MRI in 107 patients with liver disease, and measured T1 relaxation time before and after enhancement as well as SI and HUI corrected by spleen and muscle. The simple linear regression model showed that all of them were correlated with the result of ICG test, but the correlation between T1 relaxation time and ICG test was better than SI and HUI. Therefore, it is considered that the parameters based on the $\mathrm{T} 1$ relaxation time are more suitable for liver function evaluation than the 
SI-based parameters.

The above conclusion is mainly due to the fact that the SI of Gd-EOB-DTPA enhanced MRI examination is not an absolute value, and is not completely linear with the concentration of the contrast agent in the liver. In addition, due to the influence of MRI technology factors, such as RF amplification, receiving coils, and imaging sequence selection of different MRI systems, the MRI signal can be changed at different points in time. In contrast, the T1 relaxation time is an absolute value that is not affected by these factors and can provide more useful information (Figure 1).

\section{Clinical Factors Affecting Gd-EOB-DTPA Enhanced MRI}

More and more studies have shown that Gd-EOB-DTPA enhanced MRI can effectively evaluate liver function, but in clinical practice, it will also encounter insufficient HBP imaging caused by liver dysfunction, resulting in deviation. In this case, it is particularly important to predict the quality of HBP imaging before Gd-EOB-DTPA enhanced MRI scan.

Atsushi Higaki et al. [31] calculated the RE of HBP and correlated RE with various clinical parameters, finding that RE was affected by clinical parameters. They believed that the existence of chronic liver disease, ascites and splenomegaly, prothrombin activity(PTA), total bilirubin (TBIL), Alb, AST and cholinesterase $(\mathrm{CHE})$ were significant related with $\mathrm{RE}$, and it was more important to

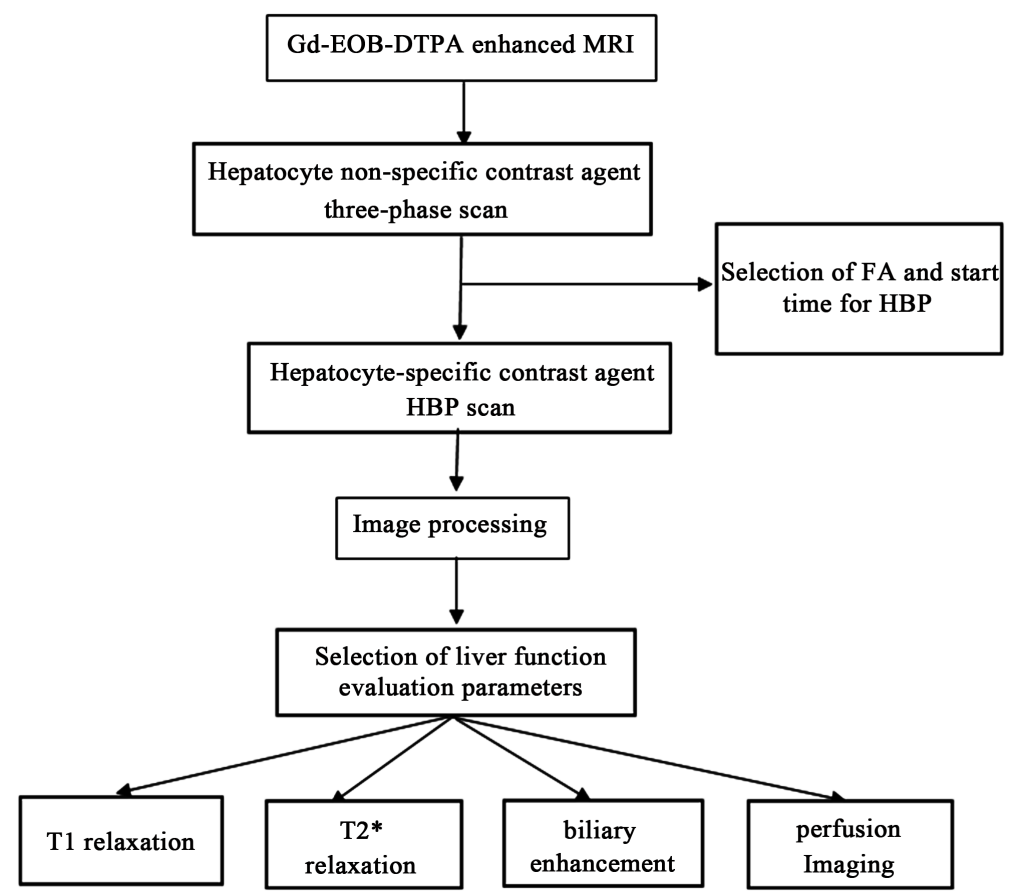

Figure 1. Regular Gd-EOB-DTPA enhanced MRI to evaluate liver function flow chart. Gd-EOB-DTPA enhanced MRI examination can be divided into two parts: hepatocyte non-specific contrast agent three-phase scan (arterial phase, portal vein phase and balance phase), hepatocyte-specific contrast agent hepatobiliary phase scan. After date collection, there're four types evaluation parameters. 
conclude that TBIL and AST were predictors of a decrease in HBP enhancement. Emina Talakic et al. [32] also reached a similar conclusion that in the hepatobiliary phase, all serum liver enzymes were significantly related with RE, in addition TBIL and CHE showed the strongest correlation. Masahiro Okada et al. [25] have found that when the activity of PT was greater than $86.9 \%$, the Gd-EOB-DTPA enhanced MRI time can be shortened. Similarly, Tobias Getzin et al. [33] discovered damaged liver parenchyma quantified with MRI correlates with histological liver damage, so hepatobiliary phase of Gd-EOB-DTPA-enhanced MRI enables non-invasive assessment of recovery from liver injury.

Therefore, M. Kobi et al. [34] hypothesized: Could clinical parameters predict insufficient hepatobiliary imaging? They collected various clinical parameters of patients who underwent Gd-EOB-DTPA-enhanced MRI within three months, including TBIL, direct bilirubin (DBIL), AST, ALT, ALP, Alb, partial thromboplastin time (aPTT) and international normalized ratio (INR). Then, they in associated them with the degree of hepatobiliary enhancement in patients, MELD, TBIL, DBIL, Alb, aPTT, and INR can be used to predict poorly performing HBP imaging with high specificity and accuracy, and DBIL is the single best predictor. Also, Wang Chen et al. [35] pointed out that the increase of TBIL and aspartate aminotransferase to platelet ratio index (APRI), the reduction of Alb may predict the lack of enhancement in the hepatobiliary phase.

\section{Summary and Outlook}

The feasibility of Gd-EOB-DTP enhanced MRI to evaluate liver function has been proved by more and more scholars [33] [36] [37]. Compared to other clinical methods, Gd-EOB-DTPA enhanced MRI can not only qualitatively and quantitatively evaluate overall liver function, but also have the ability to evaluate local segment liver function. T1 relaxation is the most widely used evaluation parameter that is effected little by MRI technology factors. Before the examination, various clinical parameters can be used to predict poorly performing hepatobiliary imaging with high specificity and accuracy to avoid invalid examination.

Studies have been performed into acute, repeated-dose, reproductive and developmental toxicity, and local tolerance, contact sensitizing, and genotoxic potential. The results showed that Gd-EOB-DTPA was well tolerated with high safety margins between the single diagnostic dose and the doses showing adverse effects in animal studies [38]. The side effects of Gd-EOB-DTPA mainly include nausea, skin vasodilation, headache, abnormal taste and pain at the injection site, mostly mild to moderate, short duration, and can be relieved without treatment. Studies have also shown that side effects occur independently of the patient's gender and age [39].

However, studies have found that in patients with liver function damage, if biliary tract disease is combined, the signal of pleural effusion or peritoneal effusion will rise after magnetic resonance imaging, thus affecting the diagnosis of 
bile leakage or biliary tract injury [40]. More importantly, the challenge is that there's no consensus on the evaluation indicators and data analysis of Gd-EOB-DTPA enhanced MRI. What's more, whether the Gd-EOB-DTPA enhanced MRI is equally accurate in accessing the different types of liver disease including hepatocellular carcinoma, liver cirrhosis etc. is not sure, there's almost none research about it. We hope that Gd-EOB-DTPA can be widely used in preoperative liver function evaluation as the research progresses further, providing more help to clinicians in preoperative assessment, surgical protocol development, and prognosis prediction.

\section{Conflicts of Interest}

The authors declare no conflicts of interest regarding the publication of this paper.

\section{References}

[1] Zheng, R., Zuo, T., Zeng, H., Zhang, S. and Chen, W. (2015) Mortality and Survival Analysis of Liver Cancer in China. Zhonghua Zhong Liu Za Zhi [Chinese Journal of Oncology], 37, 697.

[2] (2014) The Incidence and Mortality of Malignant Tumors in China in 2010. Chinese Journal of Cancer, 23, 1-10.

[3] (2014) Disodium Bismuthate Enhances Liver Intensification and Liver Function in MRI Hepatobiliary Period. Chinese Journal of Hepatobiliary Surgery, 20, 553-556.

[4] Mao, Y.L. and Tong, J.X. (2009) Progress in Evaluation of Liver Function Reserve before Liver Surgery. Chinese Journal of Clinicians (Electronic Edition), 3, 1-3.

[5] Kukuk, G.M., Schaefer, S.G., Rolf, F., Hadizadeh, D.R., Samer, E., Ulrich, S., Schild, H.H. and Willinek, W.A. (2014) Hepatobiliary Magnetic Resonance Imaging in Patients with Liver Disease: Correlation of Liver Enhancement with Biochemical Liver Function Tests. European Radiology, 24, 2482-2490.

https://doi.org/10.1007/s00330-014-3291-x

[6] Geisel, D., Raabe, P., Lüdemann, L., Malinowski, M., Stockmann, M., Seehofer, D., Pratschke, J., Hamm, B. and Denecke, T. (2017) Gd-EOB-DTPA-Enhanced MRI for Monitoring Future Liver Remnant Function after Portal Vein Embolization and Extended Hemihepatectomy: A Prospective Trial. European Radiology, 27, 1-8. https://doi.org/10.1007/s00330-016-4674-y

[7] Schuhmann-Giampieri, G., Schmitt-Willich, H., Press, W.R., Negishi, C., Weinmann, H.J. and Speck, U. (1992) Preclinical Evaluation of Gd-EOB-DTPA as A Contrast Agent in MR Imaging of the Hepatobiliary System. Radiology, 183, 59-64. https://doi.org/10.1148/radiology.183.1.1549695

[8] Chen, W. and Yu, R.S. (2015) The Application of Gd-EOB-DTPA Enhanced Magnetic Resonance Imaging in Hepatobiliary Diseases. Journal of Hepatobiliary and Pancreatic Surgery, 27, 467-473.

[9] Ma, K.S. and Jiang, J.Y. (2017) Application of Disodium Succinate Enhanced MRI in Precision Liver Surgery. Chinese Journal of Digestive Surgery, 16, 124-129.

[10] Dai, Y.Z. and Zeng, M.S. (2014) Clinical Application and Research of Specific Hepatobiliary Magnetic Resonance Contrast Agent Gd-EOB-DTPA in Biliary Imaging. Chinese Journal of Medical Imaging, 20, 291-294. 
[11] Xiang, C.H., Lu, W.P. and Dong, J.H. (2011) Assessment of Liver Reserve Function before Hepatectomy. Progress in Modern Chinese General Surgery, 14, 208-211.

[12] Dong, J.H., Zheng, S.S., Chen, X.P., Dou, K.F., Fan, J., Pei, P., Pei, X.P. and Lu, W.P. (2011) Expert Consensus on Liver Reserve Function Assessment before Liver Resection (2011 Edition). Chinese Journal of Digestive Surgery, 10, 20-25.

[13] Halle, B.M., Poulsen, T.D. and Pedersen, H.P. (2015) Indocyanine Green Plasma Disappearance Rate as Dynamic Liver Function Test in Critically Ill Patients. Acta Anaesthesiologica Scandinavica, 58, 1214-1219. https://doi.org/10.1111/aas.12406

[14] Sato, Y., Matsushima, S., Inaba, Y., Sano, T., Yamaura, H., Kato, M., Shimizu, Y., Senda, Y. and Ishiguchi, T. (2015) Preoperative Estimation of Future Remnant Liver Function Following Portal Vein Embolization Using Relative Enhancement on Gadoxetic Acid Disodium-Enhanced Magnetic Resonance Imaging. Korean Journal of Radiology Official Journal of the Korean Radiological Society, 16, 523-530. https://doi.org/10.3348/kjr.2015.16.3.523

[15] Sumiyoshi, T., Shima, Y., Okabayashi, T., Kozuki, A., Hata, Y., Noda, Y., Kouno, M., Miyagawa, K., Tokorodani, R. and Saisaka, Y. (2016) Liver Function Assessment Using 99mTc-GSA Single-Photon Emission Computed Tomography (SPECT)/CT Fusion Imaging Inhilar Bile Duct Cancer: Aretrospective Study. Surgery, 160, 118-126. https://doi.org/10.1016/j.surg.2016.02.0093

[16] Yoshida, M., Beppu, T., Shiraishi, S., Tsuda, N., Sakamoto, F., Okabe, H., Hayashi, H., Baba, H. and Yamashita, Y. (2015) 99mTc-GSA SPECT/CT Fused Images for Assessment of Hepatic Function and Hepatectomy Planning. Annals of Translational Medicine, 3, 17.

[17] Haimerl, M., Poelsterl, S., Beyer, L.P., Wiesinger, I., Nießen, C., Stroszczynski, C., Wiggermann, P. and Jung, E.M. (2016) Chronic Liver Disease: Quantitative MRI vs. CEUS-Based Microperfusion. Clinical Hemorheology \& Microcirculation, 64, 1-12.

[18] Zhou, Z.P., Long, L.L., Qiu, W.J., Cheng, G., Huang, L.J., Yang, T.F. and Huang, Z.K. (2017) Evaluating Segmental Liver Function Using T1 Mapping on Gd-EOB-DTPA-Enhanced MRI with a 3.0 Tesla. BMC Medical Imaging, 17, 20. https://doi.org/10.1186/s12880-017-0192-X

[19] Hamm, B., Staks, T., Mühler, A., Bollow, M., Taupitz, M., Frenzel, T., Wolf, K.J., Weinmann, H.J. and Lange, L. (1995) Phase I Clinical Evaluation of Gd-EOB-DTPA as a Hepatobiliary MR Contrast Agent: Safety, Pharmacokinetics, and MR Imaging. Radiology, 195, 785-792. https://doi.org/10.1148/radiology.195.3.7754011

[20] Agnello, F., Burgio, M.D., Picone, D., Vernuccio, F., Cabibbo, G., Giannitrapani, L., Taibbi, A., Agrusa, A., Bartolotta, T.V. and Galia, M. (2016) Magnetic Resonance Imaging of the Cirrhotic Liver in the Era of Gadoxetic Acid. World Journal of Gastroenterology, 22, 103-111. https://doi.org/10.3748/wjg.v22.i1.103

[21] Palmucci, S. (2014) Focal Liver Lesions Detection and Characterization: The Advantages of Gadoxetic Acid-Enhanced Liver MRI. World Journal of Hepatology: English (Electronic Version), 6, 477-485.

[22] Ünal, E., Akata, D. and Karcaaltincaba, M. (2016) Liver Function Assessment by Magnetic Resonance Imaging. Seminars in Ultrasound Ct \& Mri, 37, 549. https://doi.org/10.1053/j.sult.2016.08.006

[23] Zhou, Z.P., Long, L.L., Qiu, W.J., Cheng, G., Huang, L.J., Yang, T.F. and Huang, Z.K. (2017) Comparison of 10- and 20-min Hepatobiliary Phase Images on Gd-EOB-DTPA-Enhanced MRI T1 Mapping for Liver Function Assessment in Clinic. Abdominal Radiology, 42, 1-7. https://doi.org/10.1007/s00261-017-1143-2

[24] Jeon, I., Cho, E.S., Kim, J.H., Kim, D.J., Yu, J.S. and Chung, J.J. (2016) Feasibility of 
10-Minute Delayed Hepatocyte Phase Imaging Using a $30^{\circ}$ Flip Angle in Gd-EOB-DTPA-Enhanced Liver MRI for the Detection of Hepatocellular Carcinoma in Patients with Chronic Hepatitis or Cirrhosis. PLoS ONE, 11, e0167701. https://doi.org/10.1371/journal.pone.0167701

[25] Okada, M., Wakayama, T., Yada, N., Hyodo, T., Numata, K., Kagawa, Y., Nishiyama, D., Miyakoshi, K. and Murakami, T. (2014) Optimal Flip Angle of Gd-EOB-DTPA-Enhanced MRI in Patients with Hepatocellular Carcinoma and Liver Metastasis. Abdominal Imaging, 39, 694-701. https://doi.org/10.1007/s00261-014-0096-y

[26] Cui, E., Long, X., Li, Z., Luo, X., Lan, Y., Huang, L. and Hu, M. (2017) Feasibility Study of Increasing the Flip Angle to Shorten the Hepatobiliary Delay Time of Gd-EOB-DTPA. Radiology Practice, 32, 153-156.

[27] Lan, L., Qiu, L. and Shu, J. (2015) Progress in the Evaluation of Liver Reserve Function by Hepatocyte-Specific MRI Contrast Agent Disodium Citrate. Chinese Journal of Medical Imaging, 23, 632-634.

[28] Yoon, J.H., Lee, J.M., Kim, E., Okuaki, T. and Han, J.K. (2017) Quantitative Liver Function Analysis: Volumetric T1 Mapping with Fast Multisection B1 Inhomogeneity Correction in Hepatocyte-Specific Contrast-Enhanced Liver MR Imaging. Radiology, 282, 408. https://doi.org/10.1148/radiol.2016152800

[29] Ding, Y., Rao, S.X., Chen, C., Li, R. and Zeng, M.S. (2015) Assessing Liver Function in Patients with HBV-Related HCC: A Comparison of T? Mapping on Gd-EOB-DTPA-Enhanced MR Imaging with DWI. European Radiology, 25, 1392-1398. https://doi.org/10.1007/s00330-014-3542-x

[30] Haimerl, M., Schlabeck, M., Verloh, N., Zeman, F., Fellner, C., Nickel, D., Barreiros, A.P., Loss, M., Stroszczynski, C. and Wiggermann, P. (2016) Volume-Assisted Estimation of Liver Function Based on Gd-EOB-DTPA-Enhanced MR Relaxometry. European Radiology, 26, 1-9. https://doi.org/10.1007/s00330-015-3919-5

[31] Higaki, A., Tamada, T., Sone, T., Kanki, A., Sato, T., Tanimoto, D., Higashi, H. and Ito, K. (2012) Potential Clinical Factors Affecting Hepatobiliary Enhancement at Gd-EOB-DTPA-Enhanced MR Imaging. Magnetic Resonance Imaging, 30, 689-693. https://doi.org/10.1016/j.mri.2012.01.004

[32] Talakic, E., Steiner, J., Kalmar, P., Lutfi, A., Quehenberger, F., Reiter, U., Fuchsjäger, M. and Schöllnast, H. (2014) Gd-EOB-DTPA Enhanced MRI of the Liver: Correlation of Relative Hepatic Enhancement, Relative Renal Enhancement, and Liver to Kidneys Enhancement Ratio with Serum Hepatic Enzyme Levels and eGFR. European Journal of Radiology, 83, 607-611. https://doi.org/10.1016/j.ejrad.2013.12.010

[33] Getzin, T., Gueler, F., Hartleben, B., Gutberlet, M., Thorenz, A., Chen, R., Meier, M., Bräsen, J.H., Derlin, T. and Hartung, D. (2018) Gd-EOB-DTPA-Enhanced MRI for Quantitative Assessment of Liver Organ Damage after Partial Hepatic Ischaemia Reperfusion Injury: Correlation with Histology and Serum Biomarkers of Liver Cell Injury. European Radiology, 1-10. https://doi.org/10.1007/s00330-018-5380-8

[34] Kobi, M., Paroder, V., Flusberg, M., Rozenblit, A.M. and Chernyak, V. (2017) Limitations of GD-EOB-DTPA-Enhanced MRI: Can Clinical Parameters Predict Suboptimal Hepatobiliary Phase? Clinical Radiology, 72, 55.

https://doi.org/10.1016/j.crad.2016.10.004

[35] Wang, C., Wang, Z., Ding, W., Zeng, M. and Rao, S. (2018) Gd-EOB-DTPA Enhances the Correlation between the Degree of Hepatic Parenchymal Enhancement of Liver and Gallbladder Phase and Clinical Laboratory Indicators. Journal of Clinical Radiology, 1, 66-69. 
[36] Haimerl, M., Probst, U., Poelsterl, S., Beyer, L., Fellner, C., Selgrad, M., Hornung, M., Stroszczynski, C. and Wiggermann, P. (2018) Hepatobiliary MRI: Signal Intensity Based Assessment of Liver Function Correlated to 13 C-Methacetin Breath Test. Scientific Reports, 8, Article No. 9078. https://doi.org/10.1038/s41598-018-27401-5

[37] Zhang, W., Xiao, W., Miao, Y., Hu, C. and Zhao, W. (2018) Liver Function Correlates with Liver-to-Portal Vein Contrast Ratio during the Hepatobiliary Phase with Gd-EOB-DTPA-Enhanced MR at 3 Tesla. Abdominal Radiology, 43, 2262-2269. https://doi.org/10.1007/s00261-018-1462-y

[38] Olaf, D.H., Rainer, H., Marianne, T. and Hermann, S. (2007) Preclinical Safety Evaluation of Gd-EOB-DTPA (Primovist). Investigative Radiology, 42, 830-841. https://doi.org/10.1097/RLI.0b013e318137a471

[39] Vogl, T.J., Kümmel, S., Hammerstingl, R., Schellenbeck, M., Schumacher, G., Balzer, T., Schwarz, W., Müller, P.K., Bechstein, W.O. and Mack, M.G. (1996) Liver Tumors: Comparison of MR Imaging with Gd-EOB-DTPA and Gd-DTPA. Radiology, 200, 59-67. https://doi.org/10.1148/radiology.200.1.8657946

[40] Ciolina, M., Martino, M.D., Bruno, O., Pommier, R., Vilgrain, V. and Ronot, M. (2018) Peritoneal and Pleural Fluids May Appear Hyperintense on Hepatobiliary Phase Using Hepatobiliary MR Contrast Agents. European Radiology, 28, 1-12. https://doi.org/10.1007/s00330-017-5261-6

\section{Abbreviations}

ALT: Alanine aminotransferase

AST: Aspartate aminotransferase

ALP: Alkaline phosphatase

Gd-DTPA: Gadopentetate dimeglumine

Gd-EOB-DTPA: Gadolinium ethoxybenzyl diethylenetriamine pentaacetic acid

GGT: Glutamyl transpeptidase

MRI: Magnetic resonance imaging

OATP1: Organic anion-transporting polypeptide 1

PT: Proenzyme time

The CTP classification: The Child-Turcotte-Pugh classification

The MELD score: The model for end-stage liver disease score

The ICG test: The indocyanine green test

99mTc-GSA: 99mTc Gamma Scintigraphy 